\title{
Data Driven Respiratory Signal Detection in PET Taking Advantage of Time-of-Flight Data
}

\author{
Ottavia Bertolli, Student Member, IEEE, Simon Arridge, Charles W Stearns, Fellow, IEEE, \\ Scott D Wollenweber, Senior Member, IEEE, Brian F Hutton, Senior Member, IEEE, \\ Kris Thielemans, Senior Member, IEEE
}

\begin{abstract}
Respiratory gating is a powerful tool for tackling motion-related issues in chest PET imaging. On current scanners the respiratory signal is obtained from external devices, whereas with Data-Driven methods it can be extracted directly from the data. The aim of this work is to show the increased potential of the application of Principal Component Analysis (PCA) on TOF data. We propose a methodology that retains the TOF information and compare it to the non-TOF method. We tested the method on 16 FDG oncology patients, monitored with an RPM camera. To further investigate the benefit of TOF, PCA was selectively applied to sets of TOF bins equidistant from the center. The correlation with the RPM, the level of noise and the respiratory-likeness were analysed for all the obtained respiratory signals. The results of our analysis showed that retaining the TOF information into the sinograms considerably increased the quality of the extracted respiratory signals.
\end{abstract}

Index Terms-TOF PET, respiratory motion, data driven gating, PCA.

\section{INTRODUCTION}

$\mathbf{R}$ ESPIRATORY motion during PET acquisition can lead to artefacts in the reconstructed images, leading to a loss of resolution, lowering the quantitative accuracy of this imaging technique. In order to reduce these effects, the data can be regrouped into bins with respect to the breathing state they were acquired in, decreasing the amount of motion included in each bin. This process (referred to as gating) requires a respiratory signal, which in clinical practice is obtained from external devices, whereas with Data-Driven (DD) methods (such as Principal Component Analysis, PCA) a respiratory signal can be extracted directly from the raw data, avoiding the use of external equipment [1].

With the advent of TOF PET scanners [2], more spatial information has become directly available from the raw data, and the DD methods' performance is expected to improve. In this work we propose a methodology to unlist TOF PET

This work was supported by EPSRC Industrial CASE studentship (13220093), co-sponsored by GE Healthcare, and by the National Institute for Health Research, University College London Hospitals Biomedical Research Centre.

O. Bertolli, K. Thielemans and B. F. Hutton are with the Institute of Nuclear Medicine, UCLH, 235 Euston Road (T-5), London, NW1 2BU, UK email: ottavia.bertolli.13@ucl.ac.uk

S. Arridge is with the Centre for Medical Image Computing, University College London, Gower Street, London, WC1E 6BT, UK

B. Hutton is also with the Centre for Medical Radiation Physics, University of Wollongong, Australia

C. W. Stearns and S. D. Wollenweber are with GE Healthcare, Waukesha, WI, USA.

Manuscript received November 30, 2016. listmode data into 5 dimensional sinograms, and apply PCA in order to obtain a respiratory signal. Respiratory signals are obtained from non-TOF and TOF sinograms, and also from selected parts of the TOF data. The obtained signals are then compared to an external device output.

\section{Methods}

In order to apply DD methods on the raw data, the acquired listmode files need to be unlisted into sinograms. This process bins together all the events that were acquired within a determined time interval into the corresponding time frame. With TOF data, for each event additional information is available: the time of flight evaluated with respect to the center of the Field of View (FOV), such that the events included in each time frame can be distinguished from one another by their TOF value. In our implementation of the unlisting process, the events are split into the $500 \mathrm{~ms}$ time frames, and subsequently into a determined number of TOF bins, and then dimensionally downsampled. The end result is a 5 dimensional sinogram, given by a 3D sinogram for each TOF bin of each time frame. The 5D TOF sinograms are then given as an input to PCA, that is applied as described in [3]. This implementation differs from that in [4], where Fourier Rebinning for TOF data (FORET) is applied to obtain non-TOF sinograms before using PCA.

The respiratory-likeness of the signals obtained from PCA is estimated as follows: the power spectrum of the signals is evaluated, and for each of them the ratio of the maximum power in the respiratory frequency band $([0.1,0.4] \mathrm{Hz})$ over the mean power above $0.4 \mathrm{~Hz}$ is computed and referred to as $R$ :

$$
R=\frac{\text { maximum power in }[0.1,0.4] \mathrm{Hz}}{\text { mean above } 0.4 \mathrm{~Hz}}
$$

The signal with the highest $R$ is chosen and the mean above the respiratory band gives an indication of the level of noise in the signal. As a first step, this process is applied to all data (unlisted to non-TOF and TOF sinograms), and the obtained signals are compared to the external device by evaluating the Pearson correlation.

In the second part of this work the possibility of extracting signals from specific regions of TOF-data is analyzed. As respiratory motion does not equally affect all areas of the body, different parts of the acquired data (e.g. those corresponding to the center of the body or to the arms) are expected to contribute to the respiratory signal to different extents. The TOF sinograms allow us to distinguish between events that 
were generated in different regions of the FOV, therefore selectively applying PCA to sets of TOF bins has the potential to produce respiratory signals closely related to specific areas. We applied PCA on the central TOF bin (corresponding to the area closer to the center of the FOV) and to pairs of TOF bins that correspond to areas that are equidistant from the center (see Figure 1), and compared the signals in terms of $R$ values, noise levels and correlation with the external device.

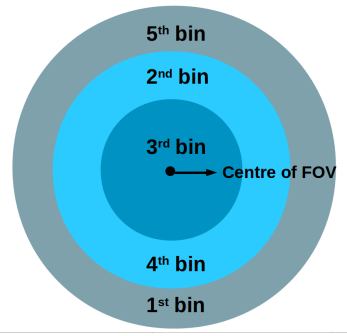

Fig. 1: Representation of 5 TOF bins in the transaxial plane. The $2^{\text {nd }}$ and $4^{\text {th }}$ and $1^{\text {st }}$ and $5^{\text {th }}$ bins correspond to the same distance from the origin.

\section{RESULTS}

We have tested our methods on 16 FDG oncology patient datasets acquired in 3D listmode on a GE Discovery 690 PET/CT scanner [5], using activity levels according to routine clinical protocols. The acquisitions were monitored by the Varian ${ }^{\circledR}$ Real-time Position Management ${ }^{\mathrm{TM}}$ (RPM) device, whose signal was utilised for comparison. The TOF resolution of the scanner is 550ps [5], and data were acquired in 55 TOF bins, of $89 \mathrm{ps}$ width. The listmode files were unlisted to a time resolution of $500 \mathrm{~ms}$ in non-TOF sinograms, and also in TOFsinograms with a total number of TOF bins of 5 or 11 (in which case, 11 and 5 TOF bins respectively were summed together).

The results of the application of PCA on the whole datasets of non-TOF and 5 and 11 TOF bins sinograms are shown in Table I. Figure 2 shows the correlations with the RPM for each patient for the three groups of sinograms, and Figure 3 shows the comparison between the respiratory signals extracted from the non-TOF, 5 and 11 TOF bins sinograms for patient 10 , where the TOF related signals showed considerably higher correlation with the RPM.

PCA was then applied to specific subsets of the TOF data. For instance, for the 5 TOF bins case the bins corresponding to equidistant areas from the center of the FOV are the following pairs: $\left(1^{\text {st }}, 5^{\text {th }}\right)$ and $\left(2^{\text {nd }}, 4^{\text {th }}\right)$ bins, and the central bin is the $3^{\text {rd }}$ one. The results of this analysis are shown in Tables II and III for the 5 and 11 TOF bins cases respectively. The correlations with RPM are shown for the 5 TOF bins case in Figure 4.

\section{Discussion}

The application of PCA on TOF sinograms provides respiratory signals that show higher correlation with the external device signal, lower level of noise and higher respiratorylikeness (represented by $R$ ), when compared to those obtained from non-TOF sinograms. The improvements resulted from
TABLE I: Mean values obtained on the 16 patients, for the $R$ value, the mean of the power spectrum above $0.4 \mathrm{~Hz}$ and the correlation with the RPM.

\begin{tabular}{l|ccc}
\multirow{2}{*}{$\mathrm{R}$} & non-TOF & 5 TOF & 11 TOF \\
\cline { 2 - 4 } Mean noise & $21 \pm 11$ & $26 \pm 11$ & $27 \pm 12$ \\
Corr with RPM & $180 \pm 62$ & $142 \pm 40$ & $124 \pm 37$ \\
\hline
\end{tabular}

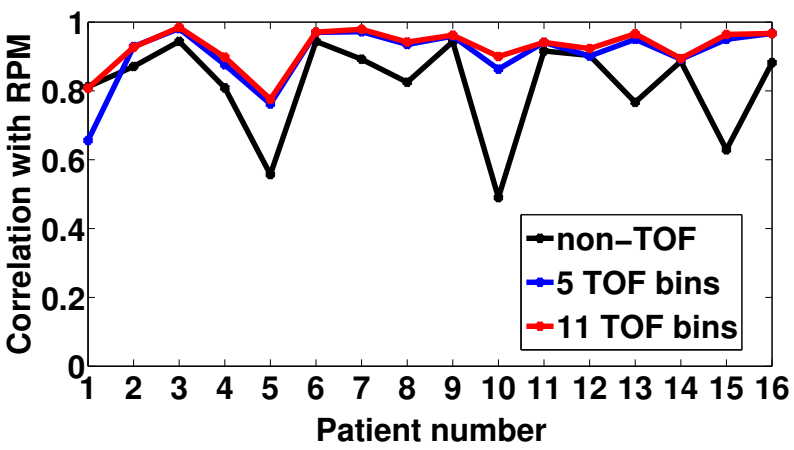

Fig. 2: Correlation with the RPM signal for all patients, for the signals obtained from non-TOF sinograms, and from 5 TOF bins and 11 TOF bins sinograms.

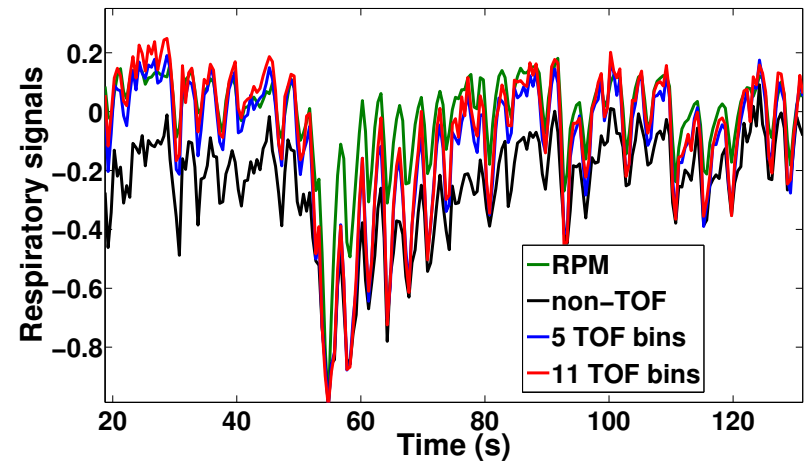

Fig. 3: Respiratory signals for patient number 10, with the RPM for comparison.

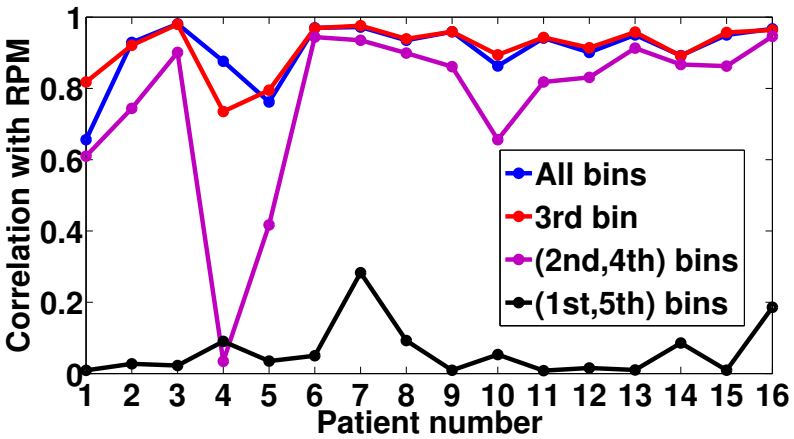

Fig. 4: Correlation with the RPM for the signals obtained by applying PCA on pairs of TOF bins, equidistant from the center of the FOV, for the 5 TOF bins case.

the use of the 5 and 11 TOF bins sinograms are comparable, suggesting that 5 TOF bins might be sufficient to efficiently exploit the TOF information contained in the data.

Moreover, when PCA is selectively applied to sets of TOF 
TABLE II: Mean values obtained on the 16 patients for the 5 TOF bins case, for the three sets of TOF bins.

\begin{tabular}{l|ccc}
\multirow{2}{*}{$\mathrm{R}$} & \multicolumn{3}{|c}{5 TOF bins } \\
\cline { 2 - 4 } Mean noise & $(1,5)$ & $(2,4)$ & 3 \\
\cline { 2 - 4 } Corr with RPM & $3.0 \pm 0.2$ & $15 \pm 8$ & $24 \pm 10$ \\
\hline
\end{tabular}

TABLE III: Mean values obtained on the 16 patients for the 11 TOF bins case, for some of the sets of TOF bins.

\begin{tabular}{l|cccc}
\multirow{2}{*}{$\mathrm{R}$} & \multicolumn{4}{|c}{11 TOF bins } \\
Mean noise & $(1,11)$ & $(3,9)$ & $(5,7)$ & 6 \\
\cline { 2 - 5 } Corr with RPM & $2.8 \pm 0.2$ & $4.5 \pm 2.6$ & $21 \pm 10$ & $22 \pm 10$ \\
\hline
\end{tabular}

bins, the data included in the central bins alone are able to provide sufficient motion information to the DD method in order to extract a respiratory signal of good quality. In Figure 4 a significant drop in the value of the correlation with RPM can be noted for patient number 4 , when using the $\left(2^{\text {nd }}, 4^{\text {th }}\right)$ pair of bins. This can be due to the lack of patient respiratory motion in the areas corresponding to the selected data.

Both for the 5 TOF and 11 TOF bin cases, processing only the data from the central bin gives a respiratory trace of nearly the same quality, as measured by the correlation with the RPM and $R$ values, compared to using all TOF bins. The small decrease in quality is likely due to increased noise when not using all of the available data. As expected, the bins corresponding to the outer regions of the FOV do not carry respiratory motion information and the corresponding signals show correlations with RPM close to zero. These TOF bins could effectively be disregarded.

\section{CONCLUSION}

The exploitation of the added spatial information conveyed by the TOF data evidently improved the performance of PCA in generating reliable respiratory signals. Moreover, unlisting TOF data into 5 dimensional TOF-sinograms provides the potential to extract the signal directly from the areas mostly affected by respiratory motion, by only considering the more central TOF bins.

\section{ACKNOWLEDGMENT}

We wish to thank François Bernard (BCCA, Vancouver, CA) for the anonymized patient data.

\section{REFERENCES}

[1] A. L. Kesner, P. J. Schleyer, F. Büther, M. A. Walter, K. P. Schäfers, and P. J. Koo, "On transcending the impasse of respiratory motion correction applications in routine clinical imaging-a consideration of a fully automated data driven motion control framework," EJNMMI Physics, vol. 1, no. 1, p. 8, 2014.

[2] S. Vandenberghe, E. Mikhaylova, E. DHoe, P. Mollet, and J. Karp, "Recent developments in time-of-flight PET," EJNMMI Physics, vol. 3, no. 1 , p. $1,2016$.
[3] K. Thielemans, S. Rathore, F. Engbrant, and P. Razifar, "Device-less gating for PET/CT using PCA," in Nuclear Science Symposium and Medical Imaging Conference (NSS/MIC). IEEE, 2011, pp. 3904-3910.

[4] M. Wang, N. Guo, H. Zhang, G. Elfhakri, G. Hu, and Q. Li, "Retrospective data-driven respiratory gating for PET using TOF information," in 2015 37th Annual International Conference of the IEEE Engineering in Medicine and Biology Society (EMBC). IEEE, 2015, pp. 4520-4523.

[5] V. Bettinardi, L. Presotto, E. Rapisarda, M. Picchio, L. Gianolli, and M. Gilardi, "Physical performance of the new hybrid PET/CT Discovery690," Medical Physics, vol. 38, no. 10, pp. 5394-5411, 2011. 